\title{
PERFORMANCE ASSESSMENT OF MOBILE RAYLEIGH DOPPLER LIDARS FOR MIDDLE ATMOSPHERE RESEARCH
}

\author{
Yuli Han', Ruocan Zhao ${ }^{1 *}$, Dongsong Sun ${ }^{1,2}$ \\ ${ }^{1}$ CAS Key Laboratory of Geospace Environment, Department of Geophysics and Planetary Sciences, \\ University of Science and Technology of China, Hefei, 230026, China, *Email: canlan@mail.ustc.edu.cn \\ ${ }^{2}$ Mengcheng National Geophysical Observatory, School of Earth and Space Sciences, University of \\ Science and Technology of China, Hefei, 230026, China
}

\begin{abstract}
Recently, two sets of mobile Rayleigh Doppler lidars were implemented in University of Science and Technology of China (USTC) for atmospheric gravity waves research. One of them works in a step stare scanning mode with azimuths corresponding to four cardinal points, while the other one consists of three fixed subassemblies: one points to the zenith and the two others are titled at $30^{\circ}$ from the zenith with east and north pointings, respectively. They both operate at eyesafe wavelength $354.7 \mathrm{~nm}$ and adopt a triple Fabry-Perot interferometer (FPI) as frequency discriminator. In order to assess the performance of the Doppler lidars, comparison experiments were performed between them. Perhaps, it is the first time to make direct comparison between scanning and non-scanning Rayleigh Doppler lidars.
\end{abstract}

\section{INTRODUCTION}

Study of atmospheric gravity waves has been an intense activity in recent years because of their myriad effects and contributions to atmospheric circulation, structure, and variability. In the middle atmosphere, between $\sim 10$ and $110 \mathrm{~km}$ altitudes, one of the most intriguing aspects of the atmosphere is the role gravity waves play in influencing its state by transporting energy and momentum between widely separated regions and generating turbulence [1]. In situ, ground-based, and space-based observation instruments have contributed great to our knowledge of gravity wave scales, amplitudes, vertical propagation and seasonal and geographic variability. Now in USTC, we have developed Mie Doppler lidar, mobile Rayleigh Doppler lidar and sodium lidar, providing profiles of wind and temperature from ground up to lower thermosphere (with wind sounding gap between 60 and $80 \mathrm{~km}$ ) with 0.2-1 $\mathrm{km}$ vertical resolution and a precision of 1-10 m/s depending on altitude. Combined observations using these instruments would greatly advance our knowledge of middle atmospheric dynamics and gravity waves. In this paper, we present our first wind field measurements by combination of two sets of mobile Rayleigh Doppler lidar, as shown in Fig. 1. Meanwhile, the comparison of two lidars' data is performed to assess the reliability and the stability of them.
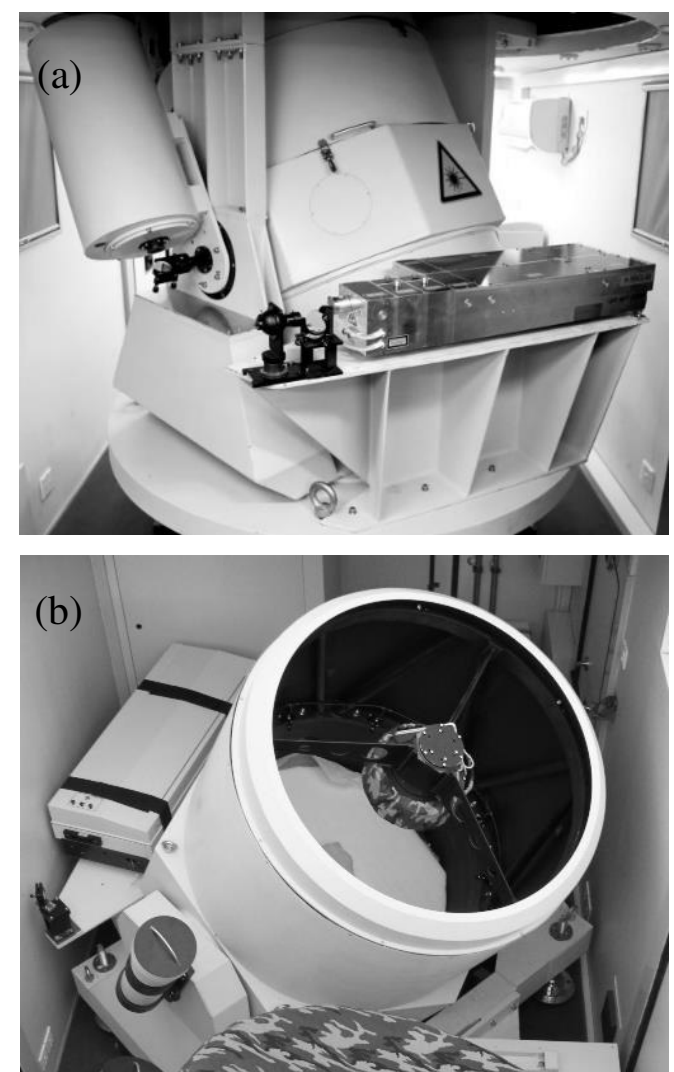

Fig. 1 Photos of the scanning (a) and non-scanning (b) mobile Rayleigh Doppler lidar system in USTC.

\section{Mobile Wind Lidar Instruments}

Two sets of mobile Rayleigh Doppler lidars are both based on the double-edge technique to realize direct-detection. The principle, optical layout, specifications, and solutions of system- 
level optical frequency stabilization for the scanning wind lidar is discussed in detail in [2]. The scanning wind lidar works in a step stare scanning mode at a fixed zenith angle of $30^{\circ}$ with azimuths corresponding to four cardinal points. Therefore the systematic error due to uncalibrated zero-wind reference can be eliminated by differential measurement of two opposite pointings. However, such superiority cannot be found in another wind lidar system, the nonscanning one. Instead of using opposite pointing to compensate the systematic error, we proposed to determine the zero wind shift by scanning the temperature-stabilized FPI with vertical atmospheric backscatter. A detailed description of the wind lidar system calibration and stabilization is presented in $[3,4]$.
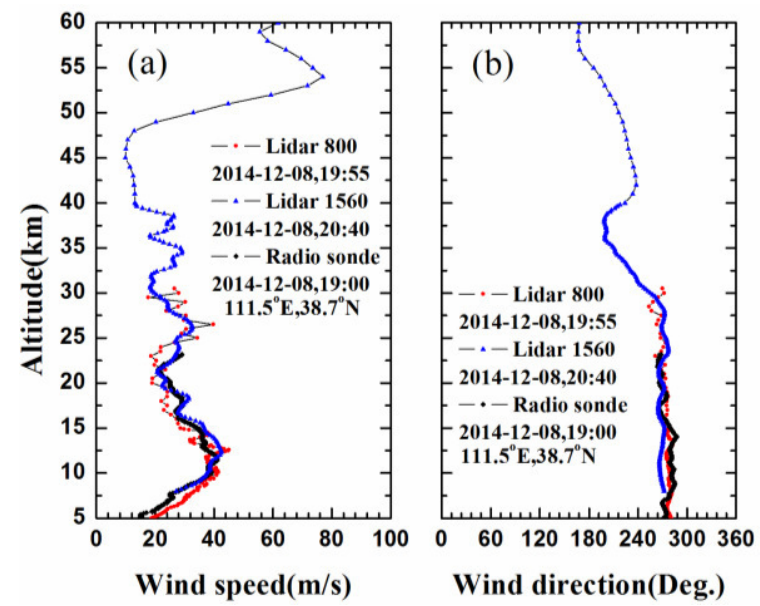

Fig. 2 Wind speed (a) and wind direction (b) of two lidars compared with radiosonde data. Lidar 800 refers to the scanning one and lidar 1560 refers to the nonscanning one.

\section{Comparison Experiments}

Observations of two lidars were performed from 6:00 pm to 7:00 am on clear weather nights. Radiosonde observations were operated twice at the same place on 7:00 pm and 7:00 am. 53 days of valid wind field data was acquired during three month of observations. Wind profiles of two lidars close to the radiosonde observation time are compared with the radiosonde data, as shown in Fig. 2. An accumulating time of $30 \mathrm{~min}$ is adopted during the wind inversion. The resolution of the scanning lidar and the non-scanning lidar changes from $100 \mathrm{~m}$ to $500 \mathrm{~m}$ at $15 \mathrm{~km}$ and from $200 \mathrm{~m}$ to $1000 \mathrm{~m}$ at $40 \mathrm{~km}$, respectively. Fig. 2 shows that the profiles of two lidars have good agreement with each other and both have good agreement with the radiosonde. It should be noted that the data is regard as invalid when the SNR (signal to noise ratio) is less than 17 . Therefore, the wind profile of the scanning lidar has valid data up to 30km in Fig. 2.

In order to have a further study of the reliability of the data at high altitude (from $30 \mathrm{~km}$ to $40 \mathrm{~km}$ ) where the signal of the scanning lidar is weak, we adjust the observation directions of two lidars to the same direction and compares the LOS (line of sight) wind velocity of two lidars at the same time, as shown in Fig. 3(a). The LOS wind profiles have good agreement below $35 \mathrm{~km}$. However, the difference between two profiles increases as altitude grows above $35 \mathrm{~km}$. This difference is caused by the weak signal of the scanning lidar above $35 \mathrm{~km}$. Fig. 3(b) shows the SNR of two lidars corresponding to these two LOS wind profiles. The SNR of the non-scanning lidar is greater than 17 below $50 \mathrm{~km}$ (see the blue dashed line). However the SNR of the scanning lidar is less than 17 above $35 \mathrm{~km}$, which is the main reason why the difference between two LOS wind profiles increases above $35 \mathrm{~km}$. Meanwhile, six continuous LOS wind profiles of two lidars are plotted together in Fig. 4 to illustrate the reliability of two lidars at different altitude. As shown in this figure, the differences of the profiles increase as SNR decreases and the detection limit of the scanning lidar is about $35 \mathrm{~km}$, which is conform with the discussion above.
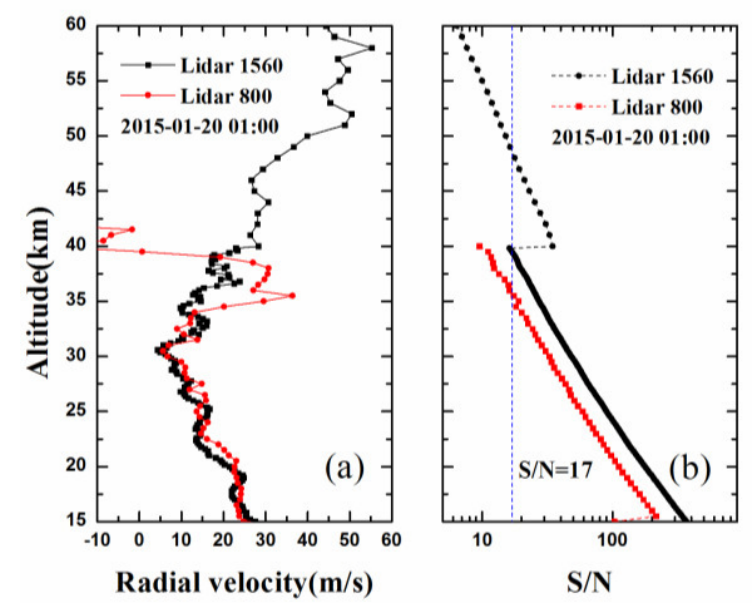

Fig. 3 (a) LOS wind velocity of two lidars (b) SNR of two lidars 


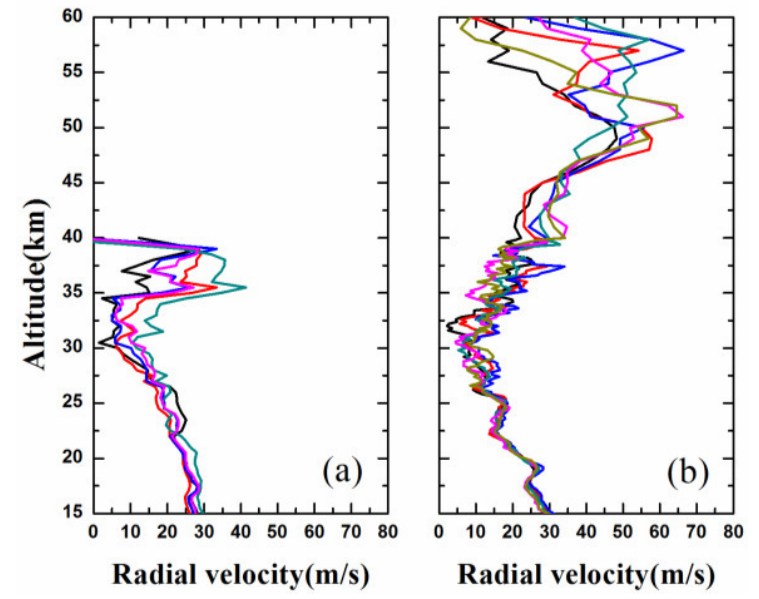

Fig. 4 Six continuous LOS wind profiles of the scanning (a) and non-scanning (b) lidar

\section{CONCLUSIONS}

Two sets of mobile Rayleigh Doppler lidars are implemented in USTC for atmospheric dynamical research. Comparison experiments are performed to assess the performance of the lidars, and the result demonstrates that their combination could provide simultaneous wind data reliable and precision enough for atmospheric gravity waves research. To fulfill the observation gap between $60 \mathrm{~km}$ to $80 \mathrm{~km}$ in the mesosphere would be our future work.

\section{ACKNOWLEDGEMENT}

This work was supported by National Natural Science Foundation of China 41174130, 41174131 and 41304123.

\section{REFERENCES}

[1] Fritts, D. C., M. J. Alexander, 2003: Gravity wave dynamics and effects in the middle atmosphere, Rev. Geophys., 40(1).

[2] Xia, H., X. Dou, D. Sun, Z. Shu, X. Xue, Y. Han, D. Hu, Y. Han, T. Cheng, 2012: Mid-altitude wind measurements with mobile Rayleigh Doppler lidar incorporating system-level optical frequency control method, Opt. Exp., 21(14), 15286-15300.

[3] Dou, X., Y. Han, D. Sun, H. Xia, Z. Shu, R. Zhao, M. Shangguan, J. Guo, 2014: Mobile Rayleigh Doppler lidar for wind and temperature measurements in the stratosphere and lower mesosphere, Opt. Exp., 22(105), A1203-A1221.

[4] Ruo-can, Z., X. Hai-Yun, D. Xian-Kang, S. Dong-Song, H. Yu-Li, S. Ming-Jia, G. Jie, S. ZhiFeng, 2015: Correction of temperature influence on the wind retrieval from a mobile Rayleigh Doppler lidar, Chin. Phys. B, 24(2), 024218. 